\title{
Comparison of Particle Simulation with J-PARC Linac MEBT Beam Test Results
}

\author{
Masanori Ikegami*, Takao Kato*, Zenei Igarashi*, Akira Ueno*, Yasuhiro Kondo ${ }^{\dagger}$, \\ Robert Ryne** and Ji Qiang**
}

\author{
${ }^{*}$ KEK, High Energy Accelerator Research Organization, 1-1 Oho, Tsukuba, Ibaraki 305-0801, Japan \\ $\dagger$ JAERI, Japan Atomic Energy Research Institute, Tokai, Naka, Ibaraki 319-1195, Japan \\ ${ }^{* *}$ LBNL, Lawrence Berkeley National Laboratory, 1 Cyclotron Road, Berkeley, CA 94720, USA
}

\begin{abstract}
The construction of the initial part of the J-PARC linac has been started at KEK for beam tests before moving to the JAERI Tokai campus, where J-PARC facility is finally to be constructed. The RFQ and MEBT (Medium Energy Beam Transport) has already been installed at KEK, and the beam test has been performed successfully. In this paper, the experimental results of the beam test are compared with simulation results with a 3D PIC (Particle-In-Cell) code, IMPACT.
\end{abstract}

\section{INTRODUCTION}

The J-PARC (Japan Proton Accelerator Research Complex) accelerator consists of a 400-MeV linac, a 3-GeV RCS (Rapid Cycling Synchrotron), and a 50-GeV synchrotron $[1,2]$. The linac is comprised of a $50-\mathrm{keV}$ negative hydrogen ion source, a $3-\mathrm{MeV} \mathrm{RFQ}$, a $50-\mathrm{MeV}$ DTL, a 190-MeV SDTL (Separate-type DTL), and a 400-MeV ACS (Annular Coupled Structure linac). The construction of the initial part of the J-PARC linac has been started at KEK to develop and establish the linac system before moving to the JAERI Tokai campus, where the J-PARC facility is finally to be constructed. The 324MHz RFQ and the MEBT (Medium Energy Beam Transport) has already been installed at KEK, and the beam test has been performed. For the details of the beam test, refer to the reference $[3,4]$. In this paper, we focus on the comparison of 3D PIC (Particle-In-Cell) simulations with the experimental results obtained with a transverse emittance monitor and wire scanners. As a simulation code, we use IMPACT [5] which is developed at LBNL.

Before moving to the description of experimental set up, we briefly review the layout of the MEBT. The MEBT has two main roles, namely, to perform transverse and longitudinal matching to the succeeding 324MHz DTL, and to chop beams to minimize the beam loss at the injection into the RCS. The schematic layout of the MEBT is shown in Fig.1. The MEBT includes eight quadrupole magnets (Q1 to Q8) for transverse matching, two 324-MHz buncher cavities for longitudinal matching, two rf deflection cavities (RFD's) and a scraper for beam chopping, and various instrumentation for beam

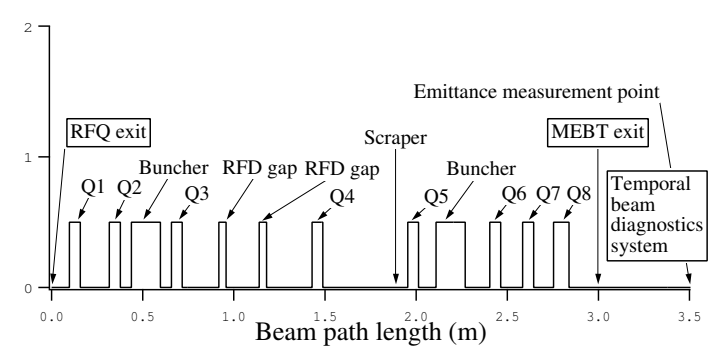

FIGURE 1. Schematic layout of the MEBT.

diagnosis. We also have five two-plane steering magnets for beam steering which are built-in to quadrupole magnets. In the measurements described in this paper, we do not perform beam chopping. The peak current of $28.7 \mathrm{~mA}$ has been achieved at the exit of the RFQ, and the transmission ratio through the MEBT reaches $99.3 \%$ without using steering magnets.

\section{EXPERIMENTAL SETUP}

In the beam test, a TBD (Temporal Beam Diagnostic system) is placed at the exit of the MEBT, which will be removed when installing the DTL. The TBD includes a transverse emittance monitor and a Faraday cup. The emittance monitor is double-slit type, and its first slit is located about $0.5 \mathrm{~m}$ downstream from the exit of the MEBT. The slit width and slit interval of the emittance monitor are $0.1 \mathrm{~mm}$ and $205 \mathrm{~mm}$, respectively.

In the actual operation, the beam should be strongly 


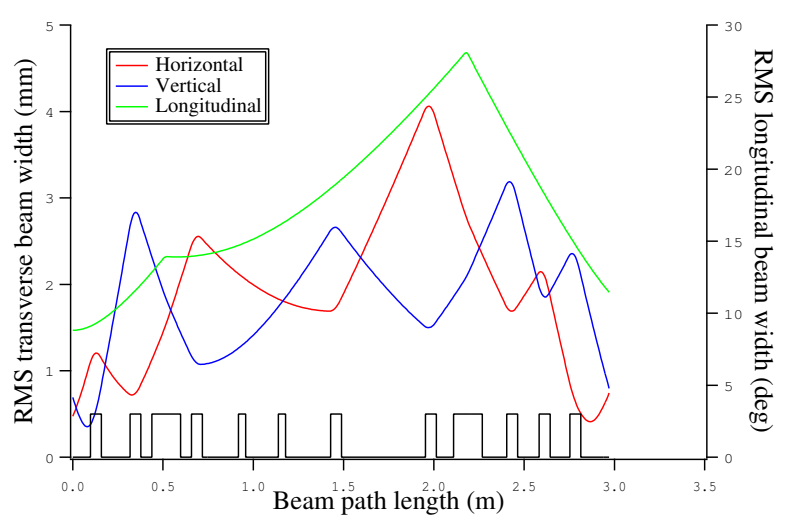

FIGURE 2. Beam envelope along the MEBT for a matched case.

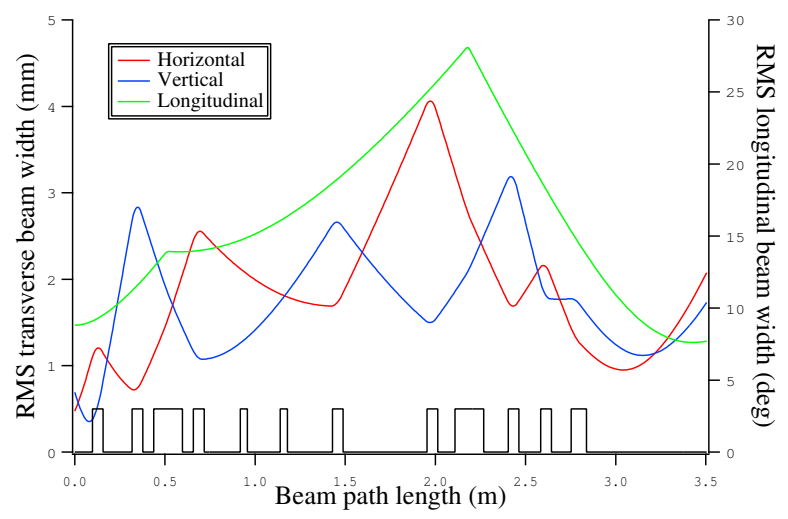

FIGURE 3. A typical beam envelope along the MEBT in the experiment.

focused at the exit of the MEBT to satisfy the matching condition to the DTL. However, the strengths of the last two quadrupoles are weakened in the experiment to enable the emittance measurement at the downstream beam diagnostic system. Figure 2 shows a beam envelope for a typical quadrupole setting which satisfies the matching condition to the DTL, and Fig. 3 shows a typical beam envelope for the experiment, in which only the last two quadrupoles are weakened. In Fig.3, the downstream end of the plot corresponds to the first slit position of the emittance monitor in the TBD. The quadrupole and buncher setting in Fig. 3 corresponds to those in the emittance measurement discussed in the next section.

In the MEBT, we have four WS's (Wire Scanners) for beam profile measurement. Each WS has horizontal, vertical and oblique (45 deg) carbon wires with the diameter of $7 \mu \mathrm{m}$. In the profile measurement, beam width is shortened to about $50 \mu \mathrm{sec}$ to reduce the heat load of the carbon wire. In the emittance measurement, we usually use the repetition ratio of $12.5 \mathrm{~Hz}$ or $25 \mathrm{~Hz}$ to shorten the measurement time, which is typically about $15 \mathrm{~min}$ for one plane.

\section{EXPERIMENTAL RESULTS}

The measured normalized rms emittances are 0.252 $\pi \mathrm{mm} \cdot \mathrm{mrad}$ and $0.214 \pi \mathrm{mm} \cdot \mathrm{mrad}$ in the horizontal and vertical planes, respectively. The emittance is measured with the emittance monitor at the TBD. The peak current in the measurement is $28.7 \mathrm{~mA}$ at the exit of the RFQ. The emittances at the exit of the RFQ was measured to be $0.173 \pi \mathrm{mm} \cdot \mathrm{mrad}$ and $0.194 \pi \mathrm{mm} \cdot \mathrm{mrad}$ in the horizontal and vertical planes, respectively, before installing the MEBT. The available beam current was, however, limited to about $10 \mathrm{~mA}$ at the time of the measurement, because the ion source has been developed in parallel with the construction. These emittances are measured with the same TBD which was placed just after the RFQ. Accordingly, we don't have measurement data for the emittance at the exit of the RFQ with the present maximum available beam current of around $29 \mathrm{~mA}$.

Figure 4 shows the phase space distribution obtained in the measurement with the TBD after the MEBT. In Fig.4, $x$ and $y$ denote the horizontal and the vertical positions, and $s$ is the path length of the design particle. Measured phase space density is represented by $100 \mathrm{k}$ dots (particles) in Fig.4 for comparison with particle simulations.

Figure 5 shows a typical beam profile measured with WS3, which is located $81 \mathrm{~mm}$ upstream from Q4. In the measurement, quadrupole setting is the same with the emittance measurement, while the buncher cavities are turned off.

\section{COMPARISON WITH SIMULATION}

As a preliminary test on the agreement between experiments and simulations, we have performed 3D PIC (Particle-In-Cell) simulations with IMPACT assuming a 6D Gaussian distribution at the exit of the RFQ. In the simulations, we assume transverse Twiss parameters at the exit of the RFQ which was obtained with the emittance measurement just after the RFQ, and the initial transverse emittances are adjusted to reproduce measured ones at the TBD after the MEBT. We also assume initial longitudinal parameters obtained with PARMTEQ[6] simulations for the RFQ. Figure 6 shows obtained phase-space distribution at the emittance monitor after the MEBT, in which we consider the same lattice setting and beam conditions with the MEBT emittance measurement. In the simulation, $1 \mathrm{M}$ simulation particles and $64 \times 64 \times 64$ meshes are employed, and 

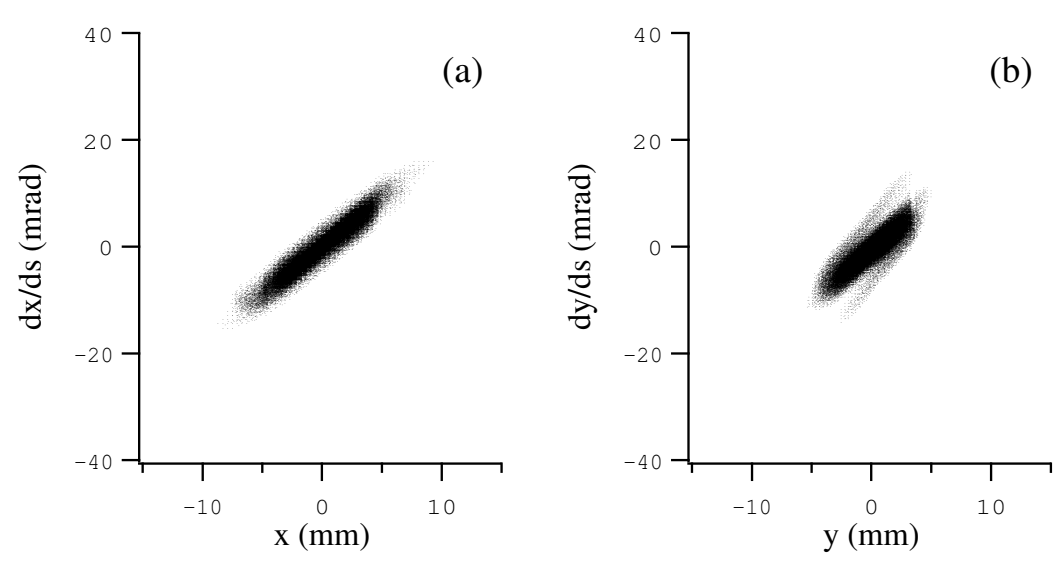

FIGURE 4. Phase-space distribution measured with the emittance monitor after the MEBT. (a) Horizontal phase plane. (b) Vertical phase plane.

the integration step width is set to $\beta \lambda / 10$ with $\beta$ and $\lambda$ being the particle velocity scaled by the speed of light and the rf wave length, respectively. The assumed initial normalized rms emittances are $0.234 \pi \mathrm{mm} \cdot \mathrm{mrad}, 0.193$ $\pi \mathrm{mm} \cdot \mathrm{mrad}$, and $0.0822 \pi \mathrm{MeV} \cdot \mathrm{deg}$ in the horizontal, vertical, and longitudinal directions, respectively. In Fig.6, $100 \mathrm{k}$ particles out of $1 \mathrm{M}$ particles are displayed. Comparing Fig. 6 with Fig.4, it is seen that the qualitative agreement between the simulation and the experiment is reasonable, while the shape of the tail portion is slightly different. In Fig.5, we show the beam profile obtained in a similar IMPACT simulation. While the simulated rms beam width is slightly wider in the horizontal direction, the agreement in the vertical direction is excellent. These agreements indicate that the tail portion is already developed to some extent at the exit of the RFQ. For comparison, we show in Fig.7 a result for waterbag case, in which we assume a 6D waterbag distribution as the initial distribution. The tail portion in the waterbag case is obviously less pronounced than in the measurement. Efforts to obtain more realistic initial distribution at the exit of the RFQ is now underway, with which the agreement between experiments and simulations is expected to be improved.

\section{SUMMARY}

The beam tests of the RFQ and the MEBT for the JPARC have been performed at KEK. The measured normalized rms emittances are $0.252 \pi \mathrm{mm} \cdot \mathrm{mrad}$ and 0.214 $\pi \mathrm{mm} \cdot \mathrm{mrad}$ in the horizontal and the vertical directions. The peak current is $28.7 \mathrm{~mA}$ at the exit of the RFQ, and the transmission ratio through the MEBT is $99.3 \%$. Preliminary simulation studies are performed with a 3D PIC code, IMPACT, and the agreement between experiment and simulation results is found reasonable in the case where a 6D Gaussian distribution is assumed at the exit of the RFQ. The agreement indicates that the tail portion is developed to some extent in the RFQ. Further study is needed to obtain more realistic distribution at the exit of the RFQ to enable quantitative prediction of tail or halo development in the downstream linac structures.

\section{ACKNOWLEDGMENTS}

The beam test has been performed by the members of J-PARC linac group. Especially, the authors would like to thank K. Nigorikawa of KEK for developing data acquisition software for the wire scanners and other beam monitors in the MEBT.

\section{REFERENCES}

1. Yamazaki, Y., "The JAERI/KEK Joint Project (the J-PARC Project) for the High Intensity Proton Accelerator," in Procs. of PAC 2003, 2003.

2. Yamazaki, Y. (ed.), Accelerator Technical Design Report for J-PARC, Tech. rep. KEK Report 2002-13; JAERI-Tech 2003-044, KEK/JAERI, (2003).

3. Ikegami, M., et. al., "Beam Commissioning of the J-PARC Linac Medium Energy Beam Transport at KEK," in Procs. of PAC 2003, 2003.

4. Kato, T., et. al., "Beam Studies with RF Choppers in the MEBT of the J-PARC Proton Linac," in Procs. of PAC 2003, 2003 .

5. Qiang, J., et. al., J. Compt. Phys., 163, 434 (2000)

6. Crandall, K. R., et. al., RFQ Design Codes, Tech. Rep. LA-UR-96-1836, LANL (1998). 

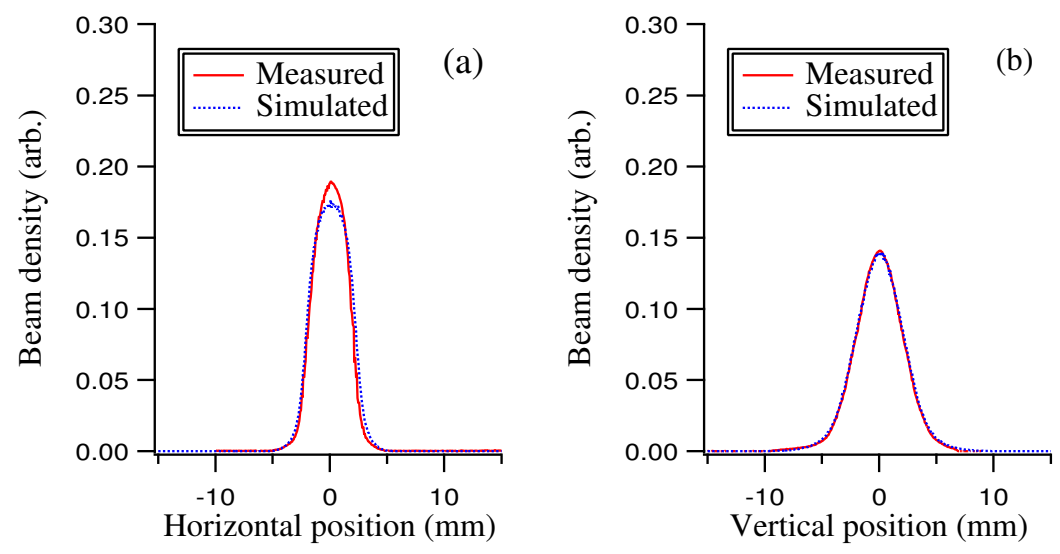

FIGURE 5. Beam profile measured with WS3 located before Q4. The quadrupole setting is the same with the emittance measurement, while the bunchers are turned off. (a) Horizontal beam profile. (b) Vertical beam profile. The beam profile obtained in an IMPACT simulation (Gaussian case) is also shown.
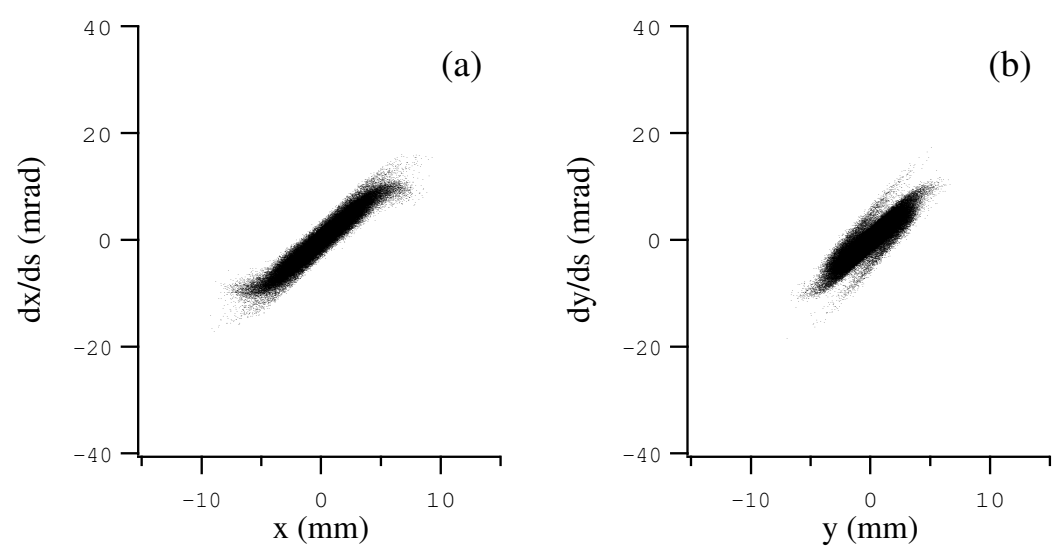

FIGURE 6. Phase-space distribution at the emittance monitor obtained with an IMPACT simulation for the emittance measurement (Gaussian case). (a) Horizontal phase plane. (b) Vertical phase plane.
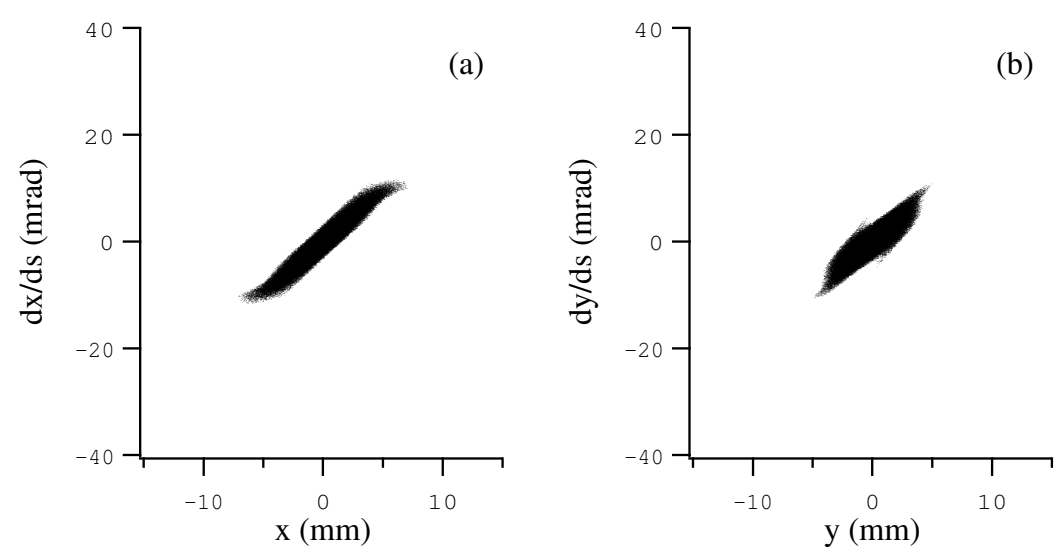

FIGURE 7. Phase-space distribution at the emittance monitor obtained with an IMPACT simulation for the emittance measurement (waterbag case). (a) Horizontal phase plane. (b) Vertical phase plane. 